\title{
Compression of superior vena cava and azygos vein by a giant mediastinal mature cystic teratoma: a case report
}

\author{
Qian Luo, Wendong Qu, Cheng Chen, Yang Liu, Yating Wei, Yongxiang Song, Xixian Ke \\ Department of Thoracic Surgery, Affiliated Hospital of Zunyi Medical University, Zunyi, China \\ Correspondence to: Dr. Prof. Yongxiang Song; Xixian Ke. Department of Thoracic Surgery, Affiliated Hospital of Zunyi Medical University, Zunyi, \\ China. Email: songtang2004@163.com; kexixian@zmu.edu.cn.
}

\begin{abstract}
Teratoma is a common type of mediastinal tumor, often located in the anterior mediastinum. Mediastinal teratomas often occur in young and middle-aged people, and account for $5-21.5 \%$ of mediastinal tumors. Mature cystic teratoma is a common type of mediastinal teratoma, its onset is slow, and most patients are asymptomatic. In a few patients, the tissue around the mediastinum is invaded or there is malignant transformation, which results in chest pain, chest tightness, a cough, and other symptoms. In this case, the patient had a giant teratoma, compressing large blood vessels and nerves, complicated by pleural and pericardial effusion. The 21-year-old female patient was misdiagnosed with tuberculous disease because of chronic cough and expectoration. Her initial symptoms improved after anti-tuberculosis treatment; however, an imaging examination showed that the lesion had enlarged some 9 months later. Surgery was performed at our hospital, as the tumor was squeezing blood vessels, and the trachea was seriously adhering to the surrounding tissue. To avoid damage to the peripheral blood vessels and nerves, many residual tissues were retained after the operation. The post-operative pathology results confirmed that the patient had a mature mediastinal cystic teratoma. One year after the operation, there was no recurrence, the peripheral blood vessels had basically returned, and the patient did not have any nerve injury. Effusion caused by mediastinal teratoma should be carefully differentiated from tuberculous diseases to avoid unnecessary damage to patients during treatment. Separation can be difficult in benign mediastinal tumors with severe adhesion. To avoid the trauma caused by the excessive separation of a tumor, it is our view that part of the residual tissue should be retained and left to be absorbed naturally.
\end{abstract}

Keywords: Mediastinal teratoma; operation; case report

Submitted Aug 04, 2021. Accepted for publication Oct 02, 2021.

doi: $10.21037 /$ tcr-21-1645

View this article at: https://dx.doi.org/10.21037/tcr-21-1645

\section{Introduction}

Mediastinal teratomas often occur in young and middleaged people, and account for $5-21.5 \%$ of mediastinal tumors (1-3). If a tumor is large, it can cause compression symptoms of the surrounding tissue. In this case, massive bleeding occurred many times during the operation because the tumor squeezed the blood vessels and trachea. Additionally, due to adhesion with the surrounding tissues, many residual tissues were retained to reduce the injury of the peripheral blood vessels and nerves. There was no sign of recurrence 1 year after the operation. The peripheral blood vessels returned, and the nerves were not damaged. We present the following article in accordance with the CARE reporting checklist (available at https://dx.doi. org/10.21037/tcr-21-1645).

\section{Case presentation}

A 21-year-old female presented at our hospital complaining of a chronic cough and coughing up sputum for 10 months. She also complained of chest tightness, shortness of breath, and pain in the right of her back when breathing hard. There was no history of similar symptoms in her 

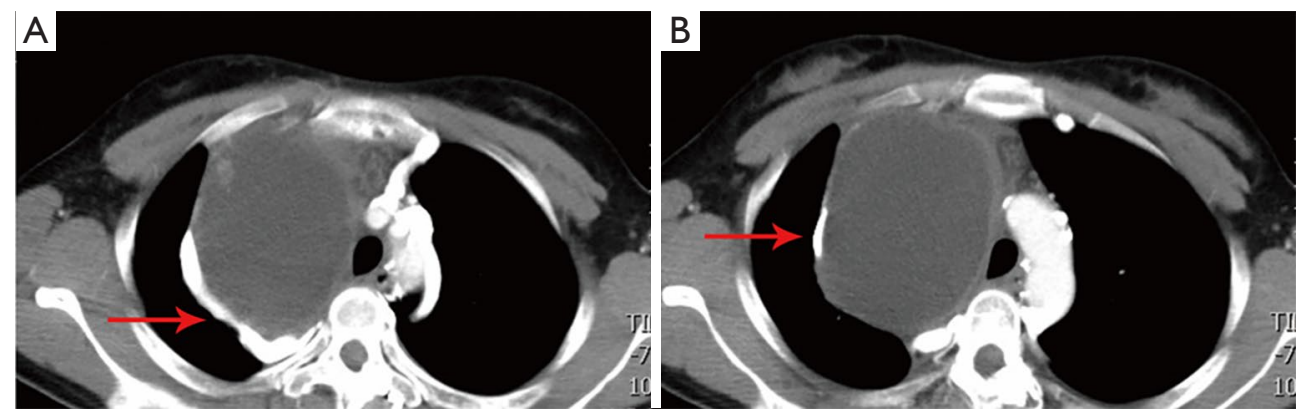

Figure 1 The pre-operative chest CT scans showed a round mass in the right middle and the superior mediastinum (A, the red arrow points to the arch of azygos vein; B, the red arrow points to the compressed superior vena cava).

family. Right tuberculous pleurisy was diagnosed after a computed tomography (CT). After treatment, the patient was discharged from the hospital and regularly took antituberculosis drugs. Nine months later, the mediastinal mass was larger, and the chest CT showed a quasi-circular water density shadow in the middle and upper mediastinum and indicated effusion in the thoracic and pericardial cavity (see Figure 1). Physical examination revealed no ptosis and the muscle strength was normal.

Tumor resection was performed in August 2018. Pleural adhesion was observed during the operation. The tumor was located in the right middle and superior mediastinum. Cystic fluid pushed outward, oppressing the deep part of the azygos vein arch and superior vena cava. The tumor reached below the right internal jugular vein, the inferior pole was located below the azygos vein arch to the hilum of the lung, and the inside the edge of the tumor reached the right internal mammary vein. The tumor surrounded the superior vena cava, phrenic nerve and azygos venous arch, and its lower part adhered severely to the hilum of the lung, the deep part of the pericardium, and the anterior mediastinum. As bleeding occurred several times during the surgery, and it was difficult to separate the tumor from normal tissues, we resected the tumor by dividing it into three parts. The first part of tumor was the triangular area formed by the superior vena cava, the azygos vein arch, and the right innominate vein. The second part was from the left side of the superior vena cava to the left edge of the tumor. The third part was from the deep part of the superior vena cava to the lower pole of the tumor. During the operation, the integrity of the tumor was destroyed as much as possible, and the surrounding nerves and blood vessels were protected (see Video S1). The postoperative pathology results confirmed a mediastinal mature cystic teratoma (see Figure 2). 1 year later, the chest CT showed that the residual tissue had been absorbed, and the blood vessels were largely restored and had healed well (see Figure 3).

All procedures performed in studies involving human participants were in accordance with the ethical standards of the institutional and/or national research committee(s) and with the Helsinki Declaration (as revised in 2013). Written informed consent was obtained from the patient for publication of this case report and accompanying images. A copy of the written consent is available for review by the editorial office of this journal.

\section{Discussion}

Mediastinal mature cystic teratoma, composed of differentiated and mature tissues, is a common type of mediastinal teratoma with slow onset and no symptoms in most patients $(4,5)$. In a few patients, the tumor invades the tissue around the mediastinum, there is malignant transformation, and other symptoms may present, such as chest pain, chest tightness, and cough $(4,5)$. If cystic teratoma of mediastinum involves the lungs, there will be recurrent pneumonia and atelectasis, and pericardial effusion may occur when pericardium is involved, invasion of bronchus may cause cough of tofu-like secretion (6). According to CT findings, most cystic teratomas are quasiround or oval, and the cavity is rich in sebum-like fluid, showing fat density or uniform liquid density, with hair and cartilage components in the capsule (7). In this case, a chest CT scan of the patient showed a round watery density shadow in the right middle and upper mediastinum, and a few small nodular and punctate density that increased the shadow at the edge, which were consistent 

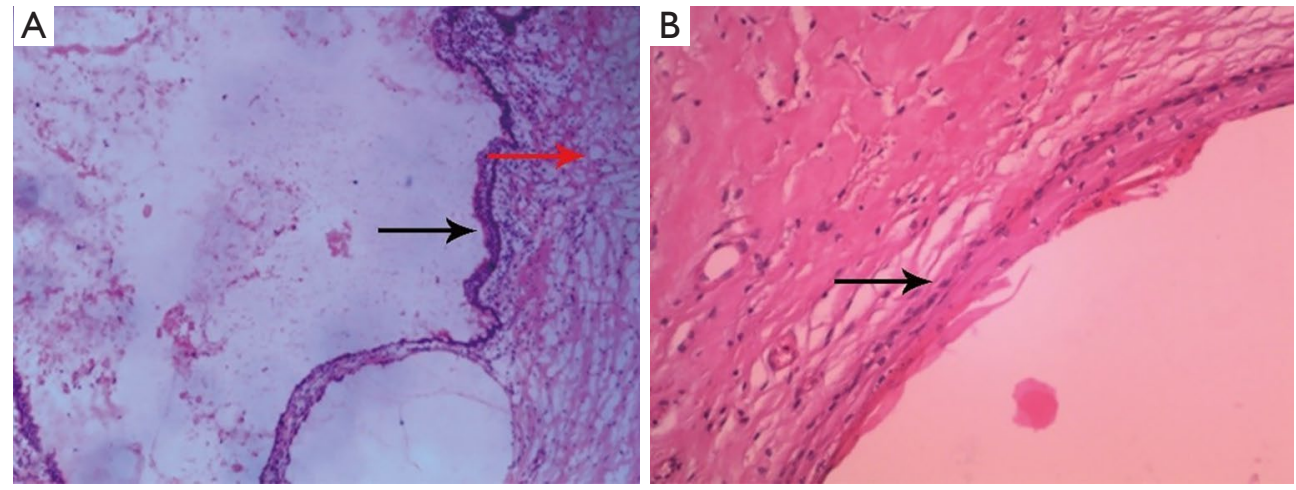

Figure 2 Post-operative pathological diagnosis of mature teratoma (HE, $\times 10)$. Mature epidermis, bronchial mucosa, and glands could be observed in the samples. (A) The black arrow points to the mucosa and the red arrow points to the acinus; (B) the black arrow points to the granular layer of epidermis.
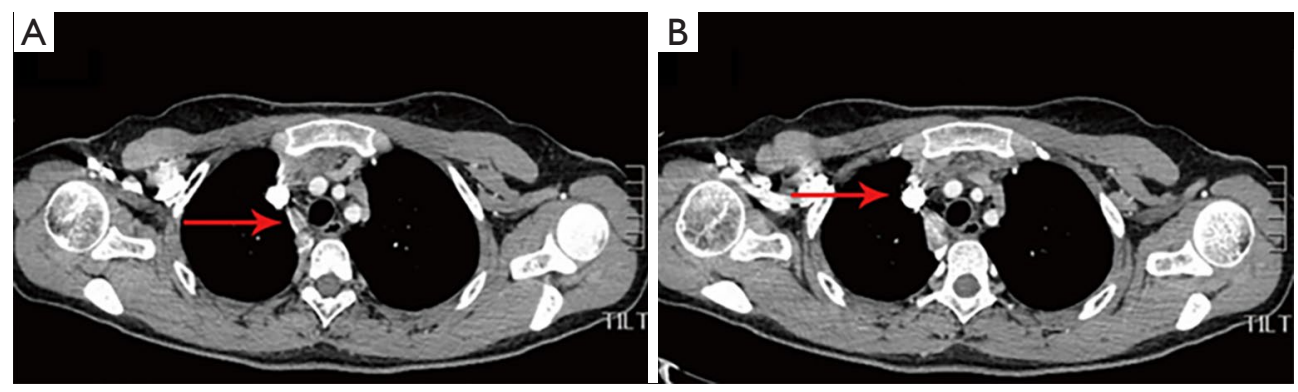

Figure 3 The chest CT scans 1 year after the operation showed that residual tissue is well absorbed (A, the red arrow points to the arch of azygos vein; B, the red arrow points to the superior vena cava).

with corresponding manifestations. For MRI, the cystic teratoma showed fat and calcified or ossified elements in the tumor (8), it is a pity that this case was not MRIexamined and therefore could not be compared.

Due to the patient's history, symptoms, and imaging examination, the case was initially misdiagnosed as tuberculous disease for a number of reasons. First, the patient's initial symptoms were cough and sputum, and an imaging examination indicated effusion in the thoracic and pericardial cavity, and the patient had lived in a region with a high incidence of tuberculosis for a long time. Second, due to the large amount of fluid contained in the cystic cavity, the imaging examination was not typical; thus, it was easy to misdiagnose it as an enveloped effusion. Third, the inexperience of the clinicians and an incomplete analysis also led to the misdiagnosis. Thus, mediastinal teratoma caused by effusion should be carefully differentiated from tuberculous diseases, and a comprehensive analysis of the clinical characteristics of the disease, combined with an auxiliary examination should be undertaken to make a clear diagnosis and avoid unnecessary damage to patients by administering diagnostic anti-tuberculous treatments. In addition, mature cystic teratoma of mediastinum rarely causes pleural effusion, but it has been reported at home and abroad that pleural effusion is the first symptom, and clinicians may be misdiagnosed as pleural effusion caused by pneumonia. In this case, there is fluid in pericardial cavity and thoracic cavity, which may be caused by the compression of superior vena cava and azygos vein $(6,9)$. Therefore, the possibility of huge mediastinal tumor should be considered when encountering recurrent pleural effusion or pericardial effusion in clinical work. Also, mediastinal cystic teratoma needs to be differentiated from pericardial cyst, bronchial cyst and other diseases.

At present, even asymptomatic mature cystic teratoma of mediastinum, complete surgical resection is always recommended, and the main method used is the thoracoscopic resection of the tumor (1). In this 
case, there was a wide range of lesions, serious adhesion between the tumor and the surrounding tissue structure, and compression. Massive bleeding occurred many times during the surgery. We were of the view that if the tumor was removed completely, it might damage the surrounding blood vessels and nerves. Thus, many residual tissues remained after operation. However, a follow-up examination showed that the residual tissues had been basically absorbed, the surrounding tissues and blood vessels were basically restored, and there was no recurrence. Thus, in our view, this approach provided an applicable and effective way to retain some of the residual tissues, which were left for natural absorption. This avoided any trauma that would have been caused by the excessive separation of the tumor. Mature teratoma of mediastinum is a benign tumor. Generally speaking, the prognosis is better after complete surgical resection. In this case, due to the serious adhesion between the tumor and the surrounding tissues, if the tumor is completely removed, it may cause fatal bleeding. Considering this, we failed to achieve the goal of completely removing the tumor, but the patient did not see tumor recurrence after CT examination one year after operation, and the residual tissues were basically absorbed. This tells us that palliative resection of benign mediastinal tumors is also acceptable. It should be noted that huge mediastinal tumor compressed superior vena cava and azygos vein, which may lead to fatal respiratory failure or circulatory failure during anesthesia. Whether percutaneous drainage of tumor contents can be performed before operation is a question worth considering. However, it is worth noting that, firstly, we can't determine whether the tumor is cystic or not and whether the contents can flow before the operation; secondly, the puncture risk is high where the tumor is located, so puncture drainage is not recommended; most importantly, if the tumor is malignant, puncture drainage may lead to tumor diffusion.

\section{Conclusions}

When a mediastinal mass was found, it should be treated surgically as soon as possible or re-examined regularly. If a large mass in the pleural cavity is found, and pleural effusion and pericardial effusion are observed, consideration should be given to a possible diagnosis of mediastinal tumor. If vascular, nerve, and severe adhesion of the benign mediastinal tumor make separation difficult, a partial resection of the tumor should be considered to protect the surrounding tissue; the residual tissue can be left to be absorbed naturally.

\section{Acknowledgments}

Funding: None.

\section{Footnote}

Reporting Checklist: The authors have completed the CARE reporting checklist. Available at https://dx.doi. org/10.21037/tcr-21-1645

Conflicts of Interest: All authors have completed the ICMJE uniform disclosure form (available at https://dx.doi. org/10.21037/tcr-21-1645). The authors have no conflicts of interest to declare.

Ethical Statement: The authors are accountable for all aspects of the work in ensuring that questions related to the accuracy or integrity of any part of the work are appropriately investigated and resolved. All procedures performed in studies involving human participants were in accordance with the ethical standards of the institutional and/or national research committee(s) and with the Helsinki Declaration (as revised in 2013). Written informed consent was obtained from the patient for publication of this case report and accompanying images. A copy of the written consent is available for review by the editorial office of this journal.

Open Access Statement: This is an Open Access article distributed in accordance with the Creative Commons Attribution-NonCommercial-NoDerivs 4.0 International License (CC BY-NC-ND 4.0), which permits the noncommercial replication and distribution of the article with the strict proviso that no changes or edits are made and the original work is properly cited (including links to both the formal publication through the relevant DOI and the license). See: https://creativecommons.org/licenses/by-nc-nd/4.0/.

\section{References}

1. Juanpere $\mathrm{S}$, Cañete $\mathrm{N}$, Ortuño $\mathrm{P}$, et al. A diagnostic approach to the mediastinal masses. Insights Imaging 2013;4:29-52.

2. Pham LH, Trinh DK, Nguyen AV, et al. Thoracoscopic surgery approach to mediastinal mature teratomas: a single-center experience. J Cardiothorac Surg 2020;15:35. 
3. Tsubochi H, Endo S, Nakano T, et al. Extraction of mediastinal teratoma contents for complete thoracoscopic resection. Asian Cardiovasc Thorac Ann 2015;23:42-5.

4. Chang CC, Chang YL, Lee JM, et al. 18 years surgical experience with mediastinal mature teratoma. J Formos Med Assoc 2010;109:287-92.

5. Takeda S, Miyoshi S, Ohta M, et al. Primary germ cell tumors in the mediastinum. Cancer 2003;97:367-76.

6. Zhan S, Zheng Y, Fan C, et al. A case of mediastinal teratoma with pleural effusion as the first manifestation. Guangdong Medical 2016;37:1081.

Cite this article as: Luo Q, Qu W, Chen C, Liu Y, Wei Y, Song $\mathrm{Y}$, Ke X. Compression of superior vena cava and azygos vein by a giant mediastinal mature cystic teratoma: a case report. Transl Cancer Res 2021;10(10):4577-4581. doi: 10.21037/ tcr-21-1645
7. Shen X, Tao J, Wang H, et al. CT diagnosis of mediastinal cystic mature teratoma. Chinese Journal of Medical Imaging 2013;21:903-6.

8. Wang Z. MRI study of mediastinal tumors and tumor-like lesions. Ningxia Medical University, 2010:12.

9. Razi E, Imani A, Ansari I, et al. Pleural effusion as a rare manifestation of mediastinal teratoma: A case report. Respir Med Case Rep 2017;22:142-4.

(English Language Editor: L. Huleatt) 
Supplementary

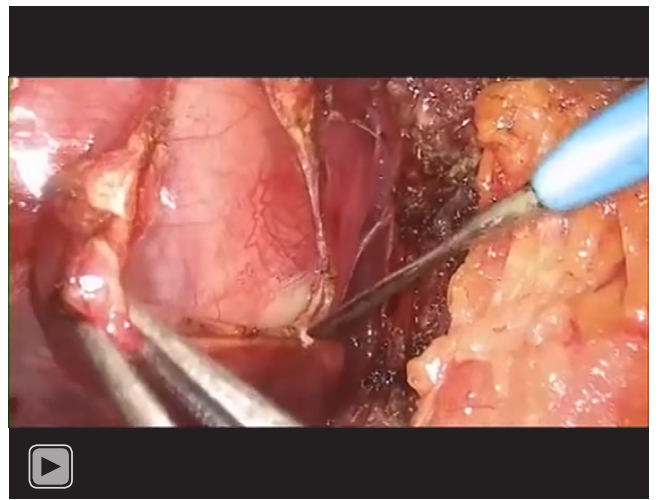

Video S1 Video of surgical resection of tumor. 